\title{
Dynamic monitoring of serum specific tumor markers predicts the response to PD-1 blockade and prognosis of patients with malignant tumors
}

\author{
Qian Dong^, Xin Sun^, Yang Zhou^, Yan-Wen Diao^, Jia-Ling Ran^, Jing-Dong Zhang^ \\ Medical Oncology Department of Gastrointestinal Tumors, Liaoning Cancer Hospital \& Institute, Cancer Hospital of China Medical University, \\ Shenyang, China \\ Contributions: (I) Conception and design: JD Zhang, Q Dong; (II) Administrative support: JD Zhang; (III) Provision of study materials or patients: Q \\ Dong; (IV) Collection and assembly of data: Q Dong, X Sun, Y Zhou, YW Diao, JL Ran; (V) Data analysis and interpretation: JD Zhang, Q Dong; (VI) \\ Manuscript writing: All authors; (VII) Final approval of manuscript: All authors. \\ Correspondence to: Jing-Dong Zhang, MD. Chief Doctor, Medical Oncology Department of Gastrointestinal Tumors, Liaoning Cancer Hospital \& \\ Institute, Cancer Hospital of China Medical University, No. 44 Xiaoheyan Road, Dadong District, Shenyang 110042, China. \\ Email: jdzhang@cancerhosp-ln-cmu.com.
}

Background: Programmed cell death protein 1 (PD-1) blockade is a major advance in the treatment of malignancies, but there remain many problems in efficacy evaluation. The aim of this study was to determine whether dynamic monitoring of serum specific tumor markers (SSTMs) could predict the response to PD-1 blockade and prognosis in patients with malignancies.

Methods: The dynamic changes in SSTMs in 27 patients between January 1, 2018 and July 31, 2019 were analyzed retrospectively. The association between the SSTM response and the radiological response was evaluated using the $\chi^{2}$ test and Spearman's correlation analysis. Kaplan-Meier estimates and the log rank test were used to explore the correlation of SSTM dynamics with progression-free survival (PFS) and overall survival (OS).

Results: In this study, $85.3 \%$ of patients with malignant tumors had detectable SSTM. According to the changes of SSTM within the first 12 weeks of treatment, the patients were divided into a tumor marker increased group and a tumor marker decreased group. The change in SSTM was strongly associated with the change in target lesions $\left(\chi^{2}=15.326, \mathrm{P} \leq 0.001\right)$, and there was a positive correlation between them (Pearson Correlation $\mathrm{r}=0.727, \mathrm{P}<0.0001)$. Patients with SSTM reduction had a significantly longer median OS (417 days) when compared with patients with SSTM elevation (median OS, 235 days; log rank $\chi^{2}=6.323$, $\mathrm{P}=0.012$ ). The PFS in the SSTM reduction group (median PFS, not reached) was significantly longer than that in the elevation group (127 days; $\log$ rank $\left.\chi^{2}=8.843, \mathrm{P}=0.003\right)$. In the study, one patient showed pseudoprogression and one showed a delayed response in the initial stage of PD-1 blockade. The diameter of the target lesions increased according to the Reaction Evaluation Criteria in Solid Tumor criteria, but the symptoms of discomfort were relieved significantly, and the SSTMs continued to decline dramatically.

Conclusions: Dynamic monitoring of SSTMs can be used as a necessary supplement to imaging examination to evaluate the response to PD-1 blockade and predict the prognosis of patients with malignancies; it may also be helpful for clinical judgment of pseudoprogression and delayed response.

Keywords: Serum specific tumor marker (SSTM); programmed cell death protein 1 (PD-1); efficacy; prognosis; malignancies

Submitted Jul 19, 2020. Accepted for publication Dec 17, 2020.

doi: $10.21037 /$ tcr-20-2560

View this article at: http://dx.doi.org/10.21037/tcr-20-2560

\footnotetext{
^ ORCID: Qian Dong, 0000-0001-9810-1368; Xin Sun, 0000-0002-2808-343X; Yang Zhou, 0000-0003-4334-6477; Yan-Wen Diao, 0000-0003-2109-2285; Jia-Ling Ran, 0000-0002-9184-3307; Jing-Dong Zhang, 0000-0002-0711-2764.
} 


\section{Introduction}

Immunotherapeutics, especially programmed cell death protein 1 (PD-1) blockade, are a major advance in the treatment of cancers. Unlike traditional cytotoxic and targeted drugs, which damage cancer cells directly, the mechanism of PD-1 blockade is to activate tumor specific lymphocytes to kill tumor cells. After starting treatment with PD-1 blockade, the immune system needs a period of time to "recruit" lymphocytes into the tumor tissue to "fight" with tumor cells (1). This may result in two forms of therapeutic response that differ from chemotherapy and targeted therapy: pseudoprogression and delayed response (2). This poses a great challenge to the traditional criteria, such as the Reaction Evaluation Criteria in Solid Tumor (RECIST) criteria, which are based on data from cytotoxic drugs used for advanced malignancies (3). Even the newly proposed modified RECIST 1.1 for immunebased therapeutics (iRECIST) (4) will face some problems in assessing the response to immunotherapy. Although iRECIST can reduce the risk of abandoning PD-1 blockade owing to mistakenly assuming ineffective treatment, it could also give rise to time being wasted on ineffective therapies that neither benefit nor are cost-effective. In addition, given the limitations of the effectiveness of PD-1 blockade monotherapy, various combined therapies are currently being applied and explored in clinical practice. The changes in the reaction kinetics of PD-1 blockade combined with chemotherapy or targeted therapy are unclear. Therefore, the evaluation method faces more challenges and this problem needs to be solved urgently.

Tumor markers are substances that are characteristic of malignant tumor cells, are abnormally produced by malignant tumor cells, or are produced by the host's stimulated response to tumors; they can reflect the occurrence and development of tumors, and be used to monitor the response to treatment $(5,6)$. Tumor markers exist in tissues, body fluids and excreta of cancer patients and can be detected by immunological, biological and chemical methods. The studies have reported that serum specific tumor markers (SSTMs) can be used to detect therapeutic effects and predict prognosis (5-8). In addition, it has been reported that the level of SSTM is closely related to tumor load, for example in the application of carcinoembryonic antigen (CEA) in colorectal cancer (9) and carbohydrate antigen 19-9 (CA19-9) in pancreatic cancer (10). Therefore, we attempted to explore whether SSTMs could be used to evaluate the efficacy of immunotherapy.
Recent studies have retrospectively analyzed the role of SSTMs in evaluating efficacy and predicting prognosis in PD-1 blockade monotherapy, mainly in non-small cell lung cancer (NSCLC). The dynamic change in cytokeratin fragment (CYFRA) 21-1 and CEA might predict the therapeutic efficacy of nivolumab in patients with advanced NSCLC (11). The higher levels of CEA were associated with inferior progression-free survival (PFS) in patients with NSCLC treated with nivolumab (12). CYFRA 21-1 has been reported to be an independent prognostic predictor for patients with advanced lung adenocarcinoma treated with nivolumab (13). Lang et al. retrospectively analyzed the association of early serum tumor marker dynamics and prognosis in patients with advanced NSCLC treated with anti-PD-1/programmed death-ligand 1 (PD-L1) therapy (14). The research showed that decreasing levels of serum tumor marker at first restaging could predict longer PFS and overall survival (OS) and identify patients with favorable prognosis among initial radiological nonresponders (14). However, the rate of effectiveness of PD-1 blocking monotherapy in most malignant tumors was less than $30 \%$, except for lymphoma, microsatellite instability-high (MSI-H) tumors, etc. At present, more efforts are being made to explore the mode of combination therapy. To date, there has been no study of the dynamic monitoring of SSTMs in evaluating the efficacy and predicting the prognosis of tumors other than NSCLC, or of the combination of PD-1 blockade with other treatment modes. We have used SSTM measurement as a supplement to imaging examination in order to evaluate the response to PD-1 blockade monotherapy and its combination with other treatment modes in clinical practice. We found that SSTMs can provide clinically relevant information when radiological examination is uncertain. Based on such observations, this study aimed to analyze retrospectively the dynamic changes of SSTMs in patients with malignancies undergoing PD-1 blockade, and to determine whether dynamic monitoring of SSTMs can be used to evaluate the therapeutic effect and predict prognosis.

We present the following article in accordance with the STROBE reporting checklist (available at http://dx.doi. org/10.21037/tcr-20-2560).

\section{Methods}

From January 2018 to July 2019, 34 consecutive patients underwent PD-1 blockade as monotherapy or in combination with other treatments as systemic treatment 
for various malignancies in our department at the Liaoning Tumor Hospital \& Institute. All of the malignancies were confirmed histologically. All patients had unresectable, recurrent or metastatic tumors. The serum tumor markers, radiological evaluation, prognosis, and other clinicopathological records of these patients were collected and retrospectively analyzed.

Among the 34 patients, two patients died of disease progression (PD) after one cycle of medication, and were excluded because of the unavailable radiological data. Five cases were excluded due to lack of elevated SSTMs before medication. They were malignant melanoma $(n=2)$, leiomyosarcoma $(n=1)$, osteosarcoma $(n=1)$, and esophageal squamous cell carcinoma $(n=1)$. Ultimately, 27 patients were included in the study. The retrospective study was conducted in accordance with the Declaration of Helsinki (as revised in 2013). The study was approved by the Research Ethics Committee of the Liaoning Tumor Hospital \& Institute (No. 2019S1103) and informed consent was taken from all the patients.

The main variables were: age, sex, types of primary tumors, treatment lines with PD-1 blockade, types of anti-PD-1 drugs, treatment regimen, SSTM level before and during treatment, and radiological response. The SSTM was defined as the serum protein tumor marker widely accepted for a certain type of malignancies in many countries, and was significantly higher than the upper limit of the reference range before the first administration of PD-1 blockade. All of the SSTM levels were measured within one week before the first administration of treatment, with an electrochemiluminescence immunoassay that used the Roche Cobas E601 (Roche, Switzerland) immunoassay system. The SSTMs of different tumors included in this study and their reference ranges are shown in Table S1. The change in SSTM from baseline was calculated using the following formula: change in SSTM from baseline = (measured SSTM level - baseline SSTM level)/baseline SSTM level. The baseline Computed Tomography (CT) or Magnetic Resonance Imaging (MRI) scans were performed before the first administration of treatment, and the following radiological evaluation was performed after every two cycles of treatment until PD. All imaging data were reviewed by two independent radiologists. The radiologists were blinded to detailed information, such as the SSTMs changes and the prognosis, but they were aware of the pathologically diagnosed malignancies. The therapeutic effect, complete response (CR), partial response $(\mathrm{PR})$, stable disease $(\mathrm{SD})$ and $\mathrm{PD}$, were evaluated on the basis of RECIST version 1.1. The change in target lesions from baseline was calculated using the following formula: change in target lesions from baseline $=$ (measured maximal diameter sum of target lesions - baseline maximal diameter sum of target lesions)/baseline maximal diameter sum of target lesions. The patients themselves or the clinicians and relatives of the patients were contacted by telephone and interviewed regarding patient survival or the documented day of death. The final survival data were collected on July $31,2019$.

\section{Statistical analyses}

The relationship between the SSTMs changes and the therapeutic effect was calculated with the $\chi^{2}$ test. Spearman's correlation analysis was performed to analyze the association between SSTM response and radiological response. Kaplan-Meier estimation was used to analyze the survival data and the statistical significance was evaluated by the log-rank test. Two-sided $\mathrm{P}$ values $<0.05$ were considered to be statistically significant. IBM SPSS Statistics version 22 and GraphPad Prism version 6.0 were used for the statistical analyses.

\section{Results}

\section{Clinicopathological and therapeutic characteristics}

Twenty-seven patients were included in this study. Table 1 lists the clinicopathological and therapeutic characteristics of the patients. The median age was 62 years $\left(\mathrm{P}_{25}-\mathrm{P}_{75}\right.$ : $59-74$ years $)$, and $20(74.1 \%)$ of them were male. All patients had unresectable, recurrent, or metastatic stage IV malignancies and were treated with PD-1 blockade as monotherapy or in combination with other treatments. Among them, 16 cases $(59.3 \%)$ were treated as first-line treatment, 5 cases $(18.5 \%)$ were treated as second-line treatment, and the other 6 cases $(22.2 \%)$ were treated as third-line or after third-line treatment. Ten cases (37\%) were of gastric and gastroesophageal junction adenocarcinoma, five hepatocellular carcinoma, five NSCLC, two esophageal squamous cell carcinoma, one small cell lung cancer, one ureteral urothelial carcinoma, one lymphoma, one hypopharyngeal squamous cell carcinoma, and one colorectal adenocarcinoma. The treatment lines and regimens for each type of tumor are detailed in Table 1. First-line treatment (59.3\%) and combination therapy $(74.1 \%)$ were most commonly used. 
Table 1 The clinicopathological and treatment characteristics of 27 patients with various cancers who received immunotherapy with anti-PD-1 monoclonal antibody

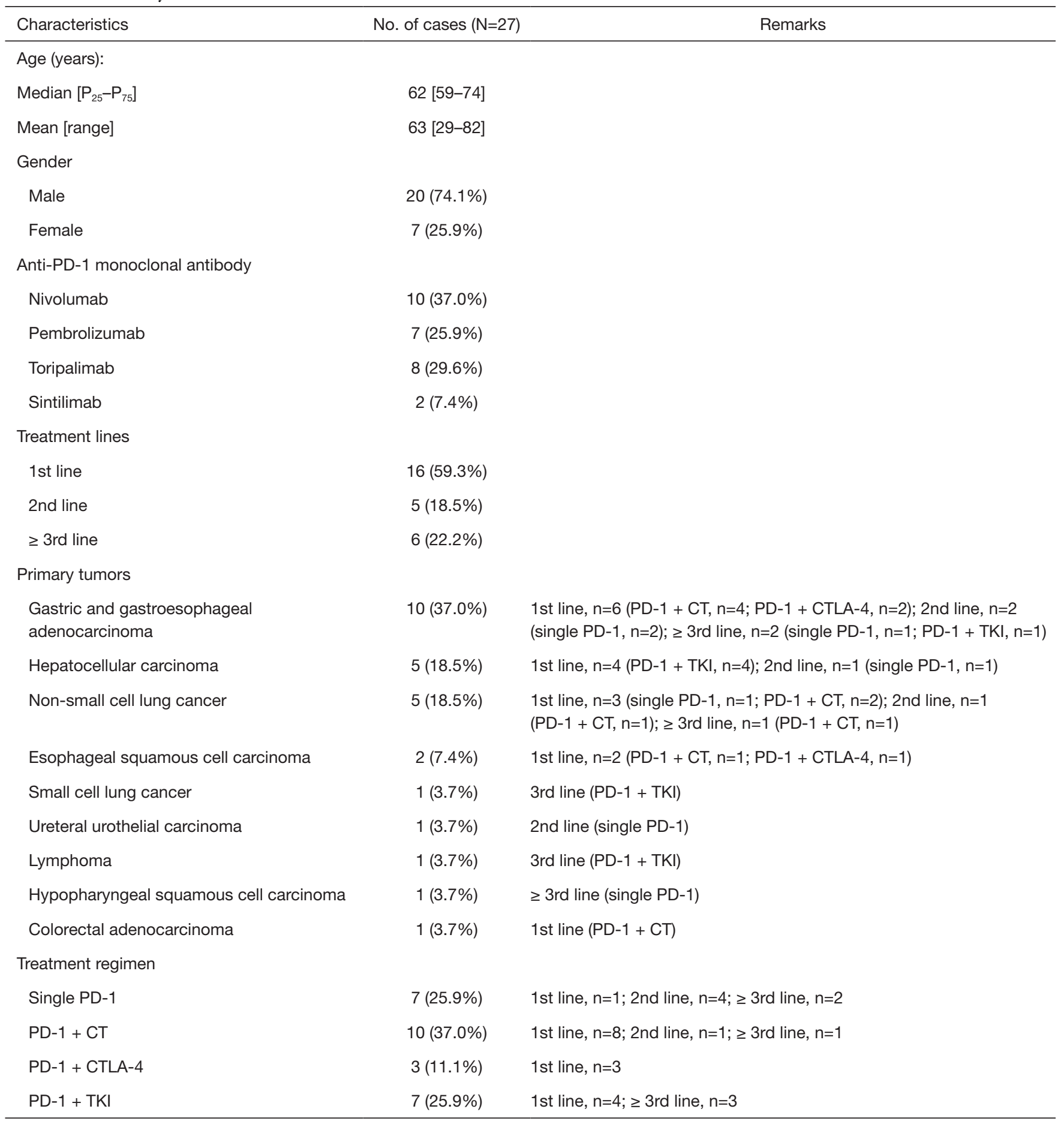

PD-1, anti-PD-1 antibody; CT, chemotherapy; CTLA-4, anti-CTLA-4 antibody; TKI, tyrosine kinase inhibitor. 

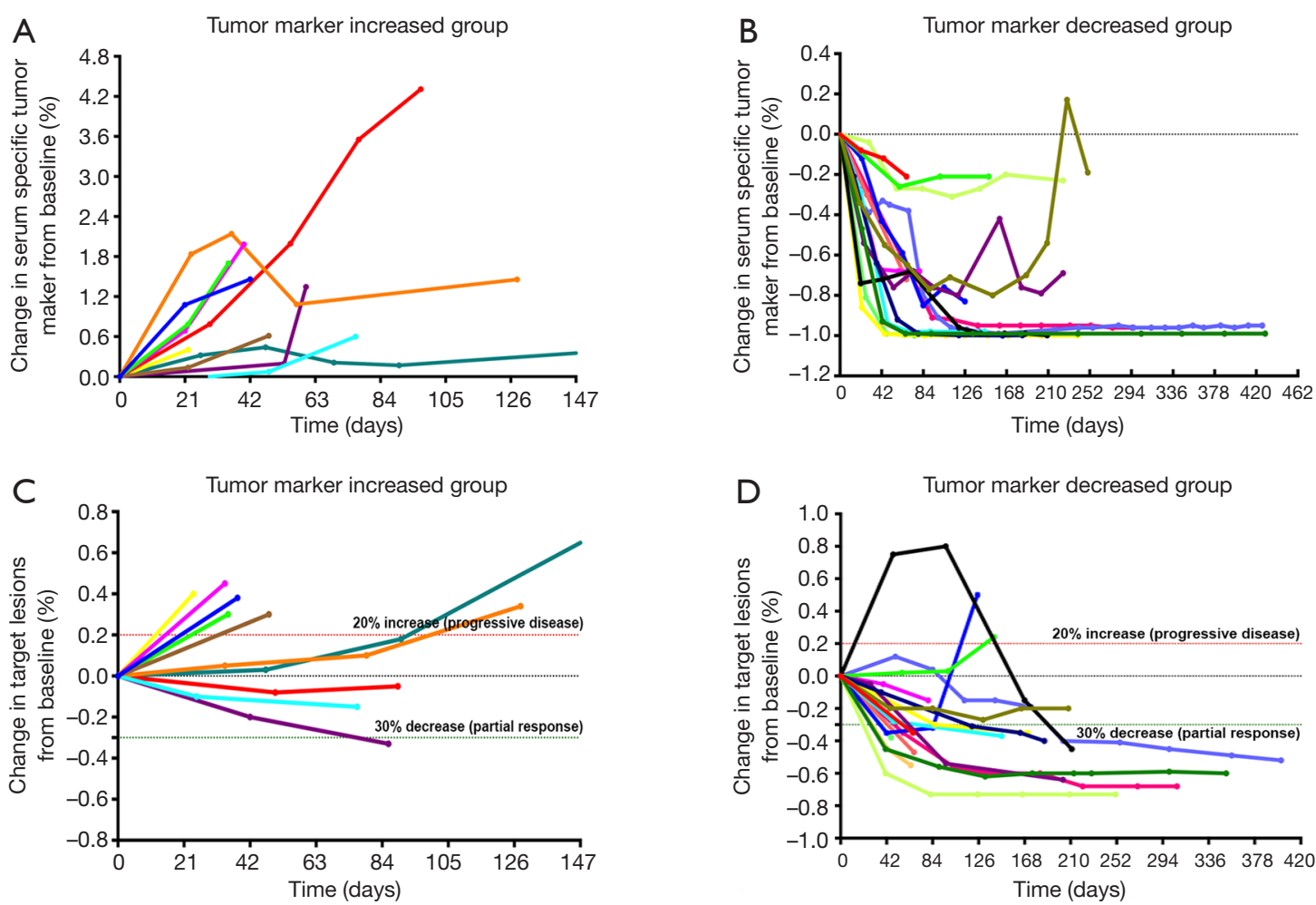

Figure 1 The changes in serum specific tumor marker (SSTM) and target lesions of each patient at different time points in the treatment process. According to the changes in SSTM within the first 12 weeks of treatment, the patients were divided into a tumor marker increased group and a tumor marker decreased group. The radiographic response was evaluated on the basis of RECIST version 1.1. (A) The SSTM response of patients in the tumor marker increased group. (B) The SSTM response of patients in the tumor marker decreased group. (C) The radiographic response of patients in the tumor marker increased group. (D) The radiographic response of patients in the tumor marker decreased group. Curves of the same color represent each patient's change. It can be seen that most of the patients with SSTM elevation in group A did not respond to treatment, and the radiographic response according to RECIST was PD; in group B, most of the patients with SSTM reduction responded to treatment, and the radiographic response was PR or SD. PR, partial response; SD, stable disease.

\section{The relationship of SSTM response and radiographic response}

The SSTM levels of the patients were measured at baseline and during the treatment. According to the SSTM changes within the first 12 weeks of treatment, the patients were divided into a tumor marker increased group (Group A) and a decreased group (Group B). The SSTM response and radiographic response of both groups are shown in Figure 1. In the trend charts of group $\mathrm{A}$ (Figure $1 A, C$ ) and group $\mathrm{B}$ (Figure 1B,D), a curve of one color represents each patient's change. It can be seen that most of the patients with SSTM elevation in group A did not respond to treatment, and the radiographic response was $\mathrm{PD}$; while in group $\mathrm{B}$, most of the patients with SSTM reduction responded to treatment, and the radiographic response was $\mathrm{PR}$ or SD. The change in SSTM was strongly associated with the change in target lesions $\left(\chi^{2}=15.326, \mathrm{P} \leq 0.001\right)$ (Table 2). Further analysis showed that the change in SSTM was positively correlated with the change in target lesions (Pearson correlation $\mathrm{r}=0.727, \mathrm{P}<0.0001$ ) (Figure 2). It should be pointed out that one patient in the group B experienced significant pseudoprogression in the initial stage of PD-1 blockade (represented by black curves in Figure 1B,D). During the whole course of treatment with anti-PD-1 antibody and Apatinib, the SSTM level continued to decline and the symptoms continued to be relieved. The black curves in Figure $1 B, D$ demonstrate the change in SSTM and radiographic response, respectively. These data suggested a strong correlation between SSTM response and 
Table 2 Changes in serum specific tumor markers within 12 weeks of the initiation of treatment with anti-PD-1 antibody, compared with radiographic response according to RECIST (one patient with false progression were removed from the analysis)

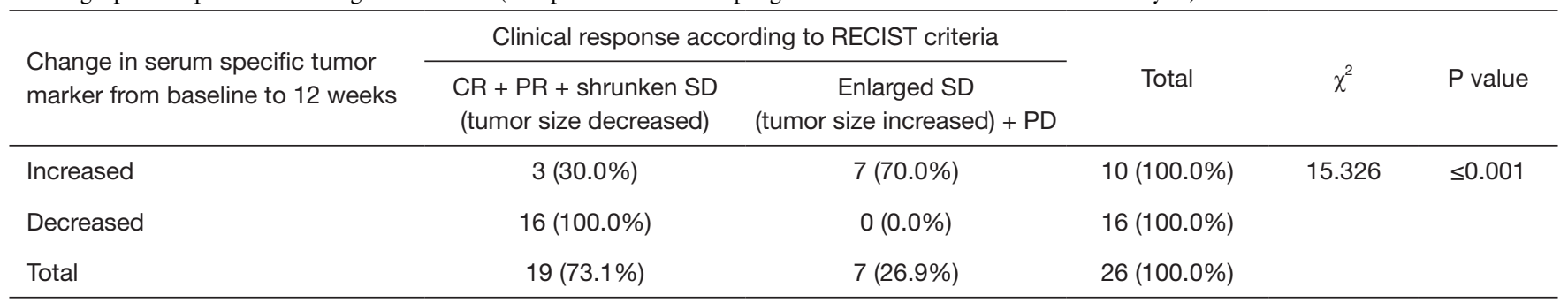

RECIST, reaction evaluation criteria in solid tumor; CR, complete response; PR, partial response; SD, stable disease; PD, disease progression.

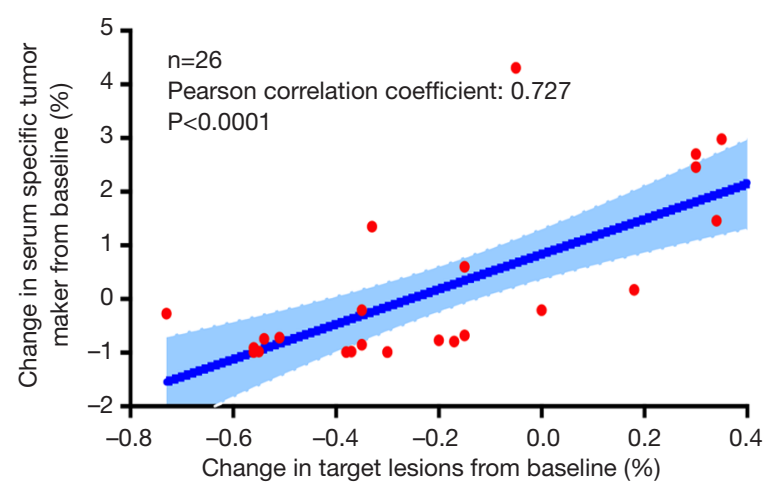

Figure 2 The change in SSTMs was positively correlated with the change in target lesions (Pearson's correlation $\mathrm{r}=0.727, \mathrm{P}<0.0001$ ). A patient with pseudoprogression was not included in the correlation analysis. SSTM, serum specific tumor marker.

radiographic response in patients receiving $\mathrm{PD}-1$ blockade, confirming that dynamic monitoring of SSTM could be used to evaluate the therapeutic response to PD-1 blockade in patients with advanced cancer, especially in cases of pseudoprogression.

\section{Evaluation of SSTM response as a prognostic predictor for patients with advanced cancer receiving PD-1 blockade}

We analyzed OS and PFS in patients with SSTM elevation and SSTM reduction. A strong correlation between SSTM response and survival time was demonstrated. Patients with SSTM reduction had a significantly longer median OS (417 days) than patients with SSTM elevation (median OS, 235 days; log-rank $\chi^{2}=6.323, \mathrm{P}=0.012$; Figure $3 A$ ). Similarly, PFS in SSTM reduction group (median PFS, not reached) was significantly longer than that in the elevation group (median PFS, 127 days; log-rank $\chi^{2}=8.843, \mathrm{P}=0.003$; Figure
3B). These data suggested that SSTM response could act as a prognostic predictor for patients with advanced cancer receiving $\mathrm{PD}-1$ blockade.

\section{SSTM may be belpful in the clinical judgment of pseudoprogression and delayed response for patients with advanced cancer receiving $P D-1$ blockade}

It is worth mentioning that there was one case of pseudoprogression and one case of delayed response in this study. The patient who experienced pseudoprogression was diagnosed with human epidermal growth factor receptor-2 (HER-2) negative alpha-fetoprotein (AFP)-producing gastric adenocarcinoma with multiple liver metastases. The patient received standard first-line and second-line chemotherapy and the disease was not controlled. Gene detection by next-generation sequencing revealed tumor mutational burden (TMB) 10.3/Mb and non-MSI-H. The patient was treated with anti-PD-1 antibody and Apatinib as third-line treatment. The diameter of the target lesions increased by $75 \%$ after two cycles of treatment and $80 \%$ after four cycles (Figure 4A). At this point the radiographic response was PD. However, abdominal pain and distension were significantly relieved, and the serum AFP decreased significantly after two cycles of treatment (Figure $4 B$ ). Considering the occurrence of pseudoprogression, it was suggested that puncture biopsy of enlarged liver metastases be performed, but the patient refused. The patient continued the treatment, and radiographic response showed shrunken SD after the 8th cycle and PR after the 10th cycle. Since then, the patient has maintained PR (Figure 4A,C). So far, throughout the treatment process with anti-PD-1 antibody and Apatinib, the serum AFP level was maintained within the reference range and with no obvious discomfort.

A patient with advanced hepatocellular carcinoma was 

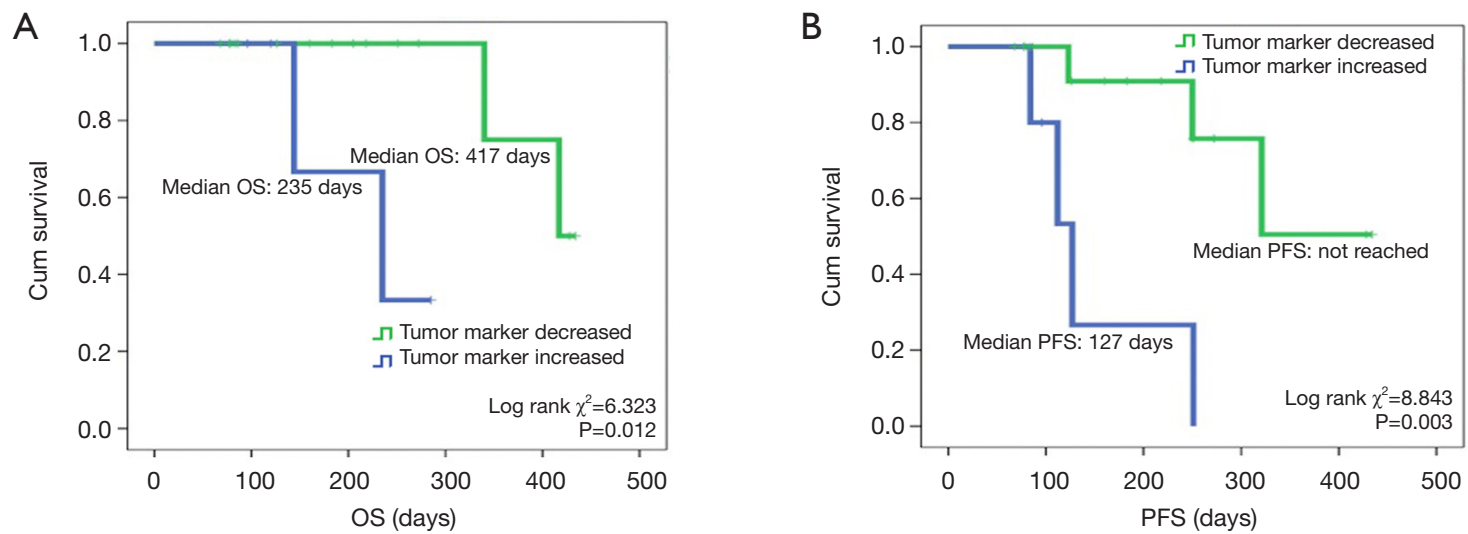

Figure 3 Comparisons of overall survival (OS) and progression-free survival (PFS) between the tumor marker increased group and tumor marker decreased group. (A) Patients with tumor marker reduction had a significantly longer median OS (417 days) compared with patients with tumor marker elevation (median OS, 235 days; log-rank $\chi^{2}=6.323, \mathrm{P}=0.012$ ). (B) PFS in the tumor marker reduction group (median PFS, not reached) was significantly longer than that in the elevation group (median PFS, 127 days; $\log -\operatorname{rank} \chi^{2}=8.843, \mathrm{P}=0.003$ ).

treated with anti-PD-1 antibody and levotinib. The patient experienced a delayed response (the maximum diameter sum of the target focus increased by $12 \%$ after 2 cycles of treatment, increased SD) and a continuous decline in serum AFP level during early evaluation. The tumor size began to decrease compared with baseline after the 6th cycle, and radiographic evaluation showed PR after the 10th cycle. The patient is still receiving treatment and maintaining PR. The change in serum AFP level and target lesions from baseline, and radiographic images, are shown in Figure 5.

These data suggested that SSTM response may be helpful for clinical judgment of pseudoprogression and delayed response for patients with advanced cancer receiving PD-1 blockade; this finding needs further discussion and analysis in a large sample of cases.

\section{Discussion}

It is suggested that SSTMs can be used to evaluate the efficacy and prognosis of chemotherapy and targeted therapy for malignant tumors. In this study, the dynamic changes of SSTMs in patients with malignancies undergoing PD-1 blockade were analyzed retrospectively. The results showed a strong correlation between SSTM response and radiographic response, and SSTM response could act as a prognostic predictor. Dynamic monitoring of SSTMs response to PD-1 blockade may be helpful for clinical judgment of pseudoprogression and delayed response.

During PD-1 blockade, there may emerge two different forms of therapeutic response: pseudoprogression and delayed response, which are different from the responses to chemotherapy and targeted therapy. For both forms of response, the application of RECIST in evaluating efficacy may lead to misjudgments. However, if the iRECIST is applied, it may lead to a failure to identify patients with true progression in time. It is important to distinguish true progression from pseudoprogression rapidly and accurately, because time should not be wasted on ineffective treatment, which is neither clinically beneficial nor costeffective. Accordingly, there is an urgent need to explore additional biomarkers for evaluating efficacy and prognostic prediction, in addition to imaging examinations, in patients with malignancies receiving PD-1 blockade as monotherapy and in combination with other treatments. Most malignancies have SSTMs, which can be easily measured in peripheral blood. In this study, $85.3 \%$ of patients with malignancies had detectable SSTMs. The SSTMs level is closely related to tumor load. The AFP level has been correlated with tumor load of hepatocellular carcinoma (15). The baseline level of CEA correlates with tumor load of metastatic colorectal cancer (9). The dynamic changes in SSTMs may indirectly reflect the changes of tumor load, so they can be used to assist in evaluating the therapeutic effect. Our study showed that dynamic change in SSTMs was positively correlated with the change in target lesions of the patients. Recent studies have reported that CYFRA 21-1 may be used to predict therapeutic efficacy (11) and be an independent prognostic predictor (13) in patients with advanced NSCLC receiving nivolumab monotherapy. CEA 
A

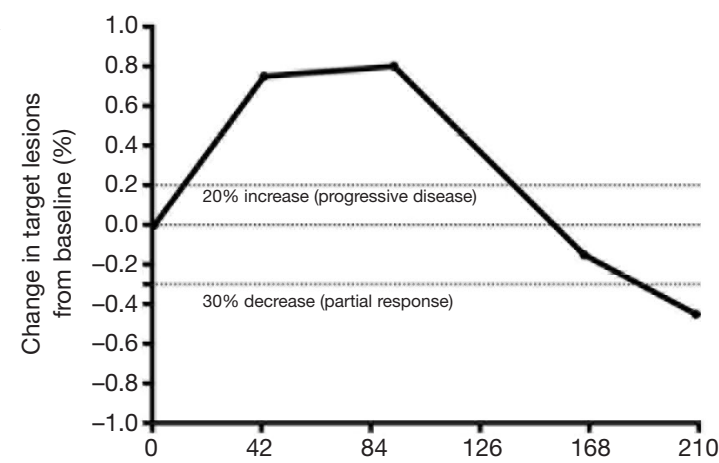

B

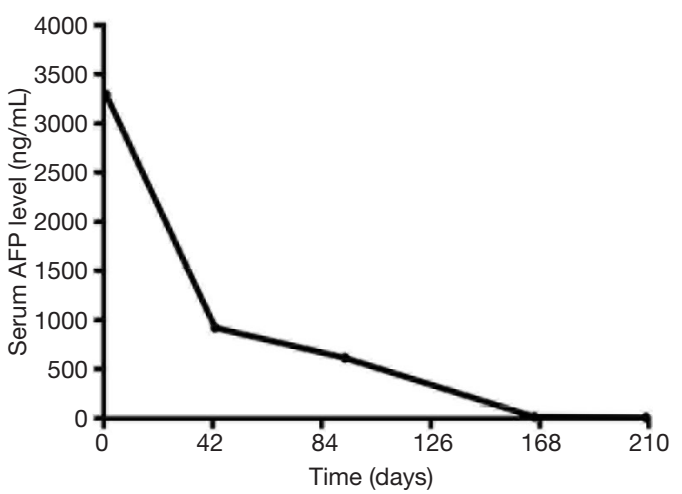

C
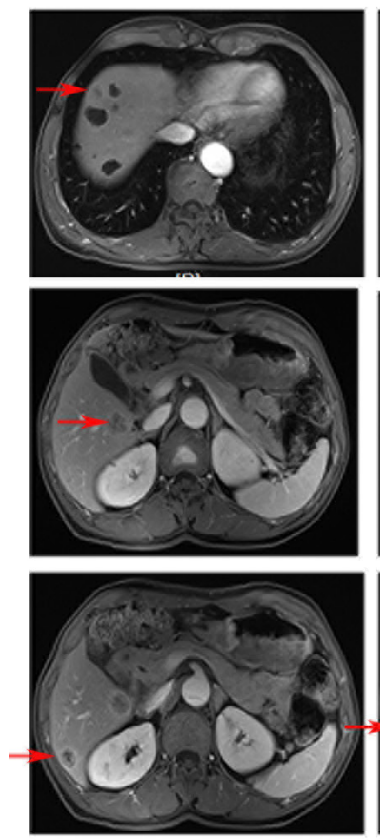

Evaluation Day 43
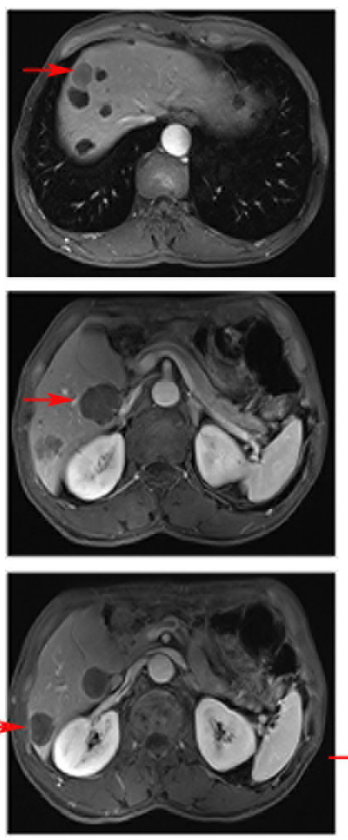

Evaluation Day 93
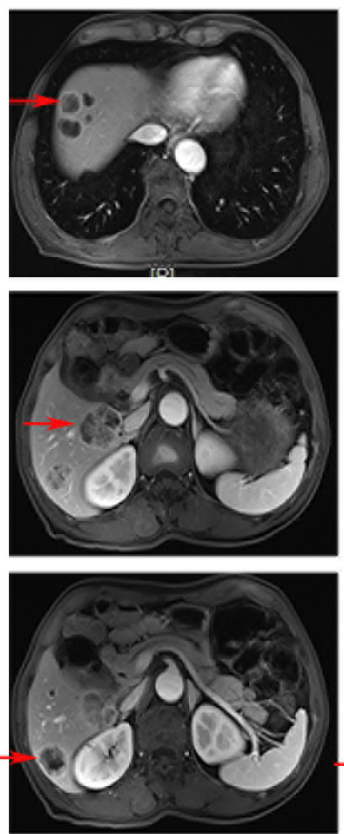

Evaluation Day 166
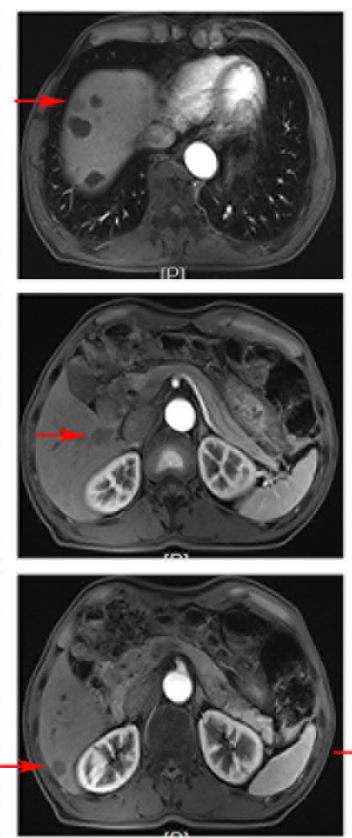

Evaluation Day 209
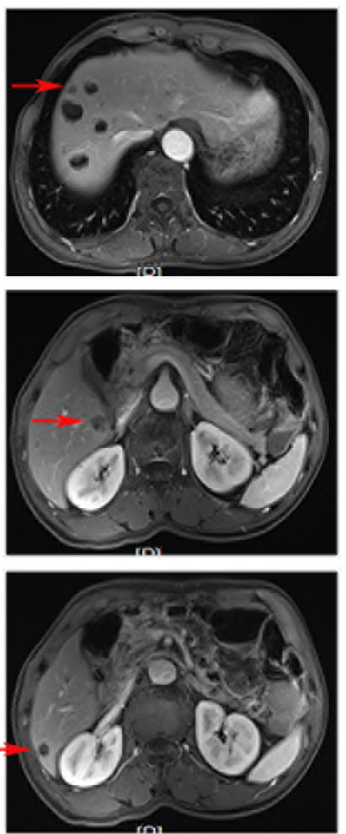

Figure 4 One patient experienced pseudoprogression of HER-2 negative AFP-producing gastric adenocarcinoma with multiple liver metastases. The patient was treated with Apatinib combined with anti-PD-1 antibody as third-line treatment. (A) The changes in target lesions evaluated on the basis of RECIST version 1.1. (B) The levels of serum AFP at different time points in the treatment process. (C) Radiographic images of liver metastases (arrows) at different time points. Anti-PD-1, anti-PD-1 antibody.

can also predict therapeutic efficacy (11) and is associated with PFS in patients with NSCLC treated with nivolumab (12). The association of early serum tumor marker dynamics and prognosis in patients with advanced NSCLC treated with PD-1/PD-L1 blockade (14). In our study, $18.5 \%$ of patients had NSCLC, and $25.9 \%$ of patients were treated with PD-1 blockade monotherapy. We have demonstrated the role of dynamic monitoring of SSTMs in all types of tumor and with various treatment modalities in this study. Attention should be paid to the patients with pseudoprogression and delayed response. At the initial stage of treatment, the diameter of target lesions was found to have increased, but the symptoms of discomfort were relieved significantly, and the SSTMs continued to decline dramatically. Pseudoprogression and delayed response are specific response modes that are observed in patients who are treated with immunotherapeutic agents $(2,16)$. The mechanism of initial progress involves infiltration of immunocytes into tumor tissues, which leads to the enlargement of tumors or new nodules. It is manifested 


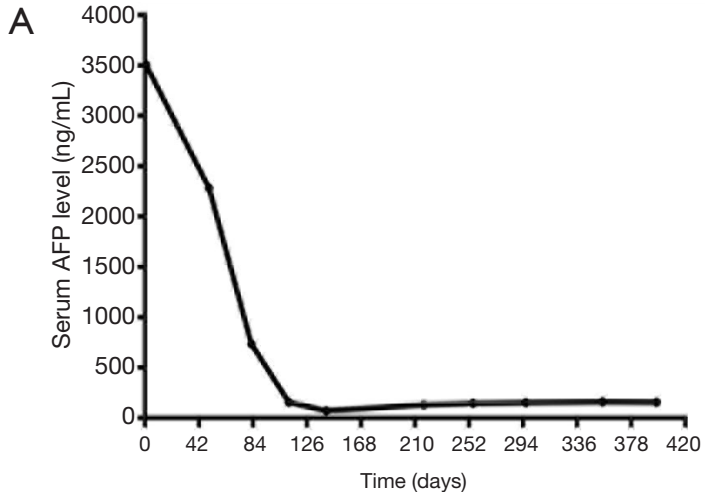

C

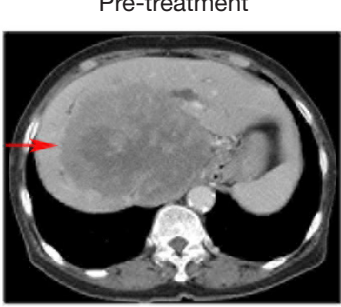

Evaluation Day 203

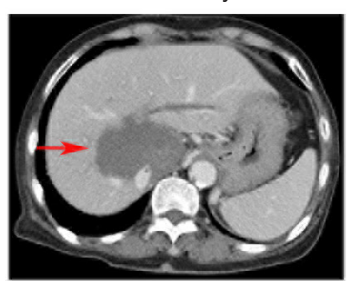

Evaluation Day 50

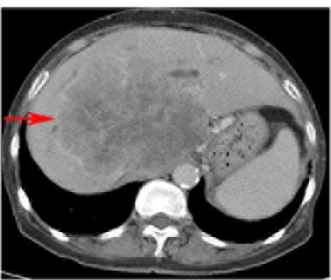

Evaluation Day 255

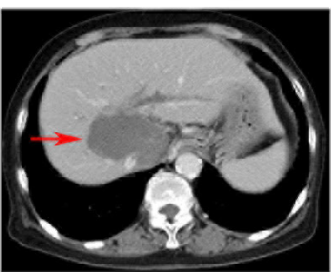

B

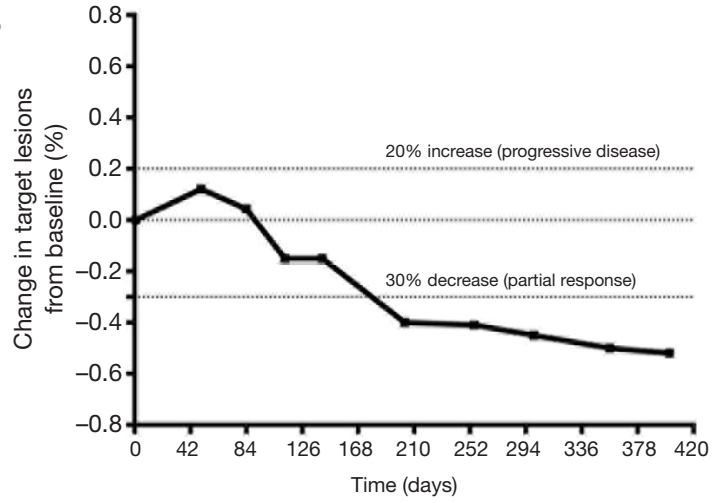

Evaluation Day 84

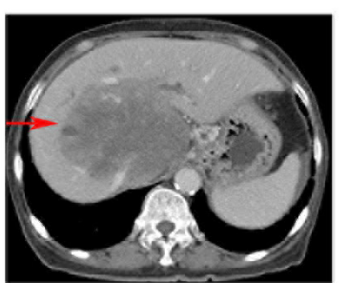

Evaluation Day 300

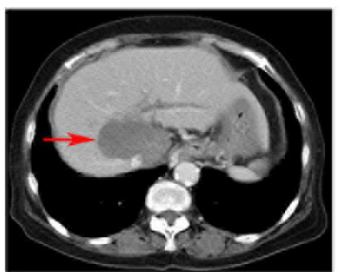

Evaluation Day 113

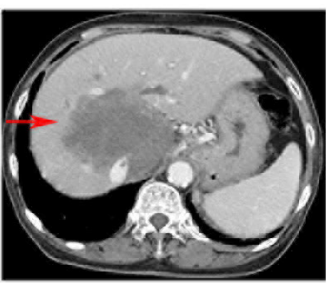

Evaluation Day 357

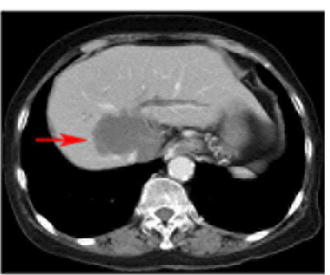

Evaluation Day 141

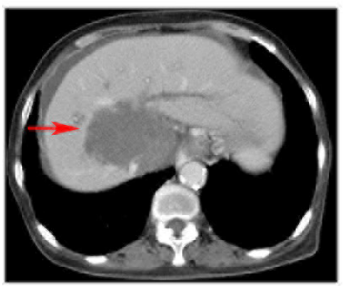

Evaluation Day 402

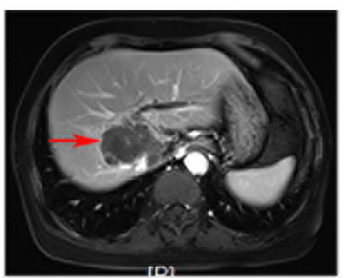

Figure 5 One patient with advanced hepatocellular carcinoma who experienced a delayed response was treated with anti-PD-1 antibody combined with levotinib from May 23, 2018. (A) Serum AFP levels at different time points in the treatment process. (B) Changes in target lesions evaluated on the basis of RECIST version 1.1. (C) Radiographic images of target lesions (arrows) at different time points. Anti-PD-1, anti-PD-1 antibody.

as imaging progression, rather than real tumor cell proliferation (16). Therefore, the SSTM level can reflect the tumor load more effectively than imaging examination at this stage.

Ideal biomarkers should be readily available, of low cost, and allow continuous detection (17). Most malignancies have SSTMs that can be easily detected in peripheral blood. It may be possible to combine the SSTMs monitoring results and imaging evaluation results to predict the therapeutic effect and prognosis of tumors. The advantages of SSTMs are convenient and economical. The advantages of imaging evaluation are universal and widely accepted. The combination of the two methods may improve the accuracy of prediction in the vast majority of cases. Only in a few cases, the two evaluation methods may be inconsistent, such as the pseudoprogression and delayed response. Because the SSTM level can reflect the tumor load more effectively than imaging examination, the SSTMs may be a useful supplement to imaging evaluation.

Lastly, there were several inevitable limitations to this retrospective study. First, the number of patients and duration of the follow-up period were major concerns, mainly because anti-PD antibody was not available in China until 2018. However, judging from the existing results, dynamic monitoring of SSTMs was indeed helpful in evaluating the efficacy of PD-1 blockade and predicting prognosis in patients with malignancies. Nevertheless, prospective validation with a sufficient number of patients is needed to clarify the clinical significance of dynamic monitoring of SSTMs. Second, we did not get enough 
pathological information from the patient who have experienced pseudoprogression, because the patient refused to accept the biopsy. However, throughout the patient's treatment process, pseudoprogression could be determined. Although there was one case of pseudoprogression and one case of delayed response, we have seen the potential value of SSTM, which needs to be verified in more cases. Last, this was a retrospective study, and there may be selective bias. In order to avoid the influence of selective bias on the results of retrospective study as much as possible, the patients that met the inclusion criteria were selected consecutively in a fixed time interval.

\section{Conclusions}

Dynamic monitoring of SSTMs can be used as a necessary supplement to imaging examination in evaluating the response to $\mathrm{PD}-1$ blockade and predicting the prognosis of patients with malignancies; it may also be helpful for clinical judgment of pseudoprogression and delayed response. These findings, if confirmed, may provide a practical method for evaluating the efficacy of immune checkpoint inhibitor therapy.

\section{Acknowledgments}

We thank International Science Editing (http://www. internationalscienceediting.com) for editing this manuscript. Funding: This work was supported by National key research and development program (No. 2018YFC1311603), the National Natural Science Foundation of China (No. 81372532), the Science and Technology Project Fund from Education Department of Liaoning Province (No. LK201619), the Science and Technology Planning Project of Liaoning Province (Nos. 201800449, 2019-ZD-1022), the Science and Technology Planning Project of Shenyang (No. F15-139-9-27), and the scientific research foundation for the introduction of talents, Liaoning Cancer Hospital \& Institute (No. Z1702).

\section{Footnote}

Reporting Checklist: The authors have completed the STROBE reporting checklist. Available at http://dx.doi. org/10.21037/tcr-20-2560

Data Sharing Statement: Available at http://dx.doi. org/10.21037/tcr-20-2560
Conflicts of Interest: All authors have completed the ICMJE uniform disclosure form (available at http://dx.doi. org/10.21037/tcr-20-2560). The authors have no conflicts of interest to declare.

Ethical Statement: The authors are accountable for all aspects of the work in ensuring that questions related to the accuracy or integrity of any part of the work are appropriately investigated and resolved. The retrospective study was conducted in accordance with the Declaration of Helsinki (as revised in 2013). The study was approved by the Research Ethics Committee of the Liaoning Tumor Hospital \& Institute (No.2019S1103) and informed consent was taken from all the patients.

Open Access Statement: This is an Open Access article distributed in accordance with the Creative Commons Attribution-NonCommercial-NoDerivs 4.0 International License (CC BY-NC-ND 4.0), which permits the noncommercial replication and distribution of the article with the strict proviso that no changes or edits are made and the original work is properly cited (including links to both the formal publication through the relevant DOI and the license). See: https://creativecommons.org/licenses/by-nc-nd/4.0/.

\section{References}

1. Wei SC, Levine JH, Cogdill AP, et al. Distinct Cellular Mechanisms Underlie Anti-CTLA-4 and Anti-PD-1 Checkpoint Blockade. Cell 2017;170:1120-33.e17.

2. Chiou VL, Burotto M. Pseudoprogression and ImmuneRelated Response in Solid Tumors. J Clin Oncol 2015;33:3541-3.

3. Schwartz LH, Litiere S, de Vries E, et al. RECIST 1.1-Update and clarification: From the RECIST committee. Eur J Cancer 2016;62:132-7.

4. Seymour L, Bogaerts J, Perrone A, et al. iRECIST: guidelines for response criteria for use in trials testing immunotherapeutics. Lancet Oncol 2017;18:e143-52.

5. Faria SC, Sagebiel T, Patnana M, et al. Tumor markers: myths and facts unfolded. Abdom Radiol (NY) 2019;44:1575-600.

6. Reiter MJ, Costello JE, Schwope RB, et al. Review of Commonly Used Serum Tumor Markers and Their Relevance for Image Interpretation. J Comput Assist Tomogr 2015;39:825-34.

7. Kirwan A, Utratna M, O'Dwyer ME, et al. GlycosylationBased Serum Biomarkers for Cancer Diagnostics and 
Prognostics. Biomed Res Int 2015;2015:490531.

8. Duffy MJ. Tumor markers in clinical practice: a review focusing on common solid cancers. Med Princ Pract 2013;22:4-11.

9. Yu P, Zhou M, Qu J, et al. The dynamic monitoring of CEA in response to chemotherapy and prognosis of mCRC patients. BMC Cancer 2018;18:1076.

10. Loosen SH, Neumann UP, Trautwein C, et al. Current and future biomarkers for pancreatic adenocarcinoma. Tumour Biol 2017;39:1010428317692231.

11. Dal Bello MG, Filiberti RA, Alama A, et al. The role of CEA, CYFRA21-1 and NSE in monitoring tumor response to Nivolumab in advanced non-small cell lung cancer (NSCLC) patients. J Transl Med 2019;17:74.

12. Kataoka Y, Hirano K, Narabayashi T, et al. Carcinoembryonic Antigen as a Predictive Biomarker of Response to Nivolumab in Non-small Cell Lung Cancer. Anticancer Res 2018;38:559-63.

13. Shirasu H, Ono A, Omae K, et al. CYFRA 21-1 predicts the efficacy of nivolumab in patients with

Cite this article as: Dong Q, Sun X, Zhou Y, Diao YW, Ran JL, Zhang JD. Dynamic monitoring of serum specific tumor markers predicts the response to PD-1 blockade and prognosis of patients with malignant tumors. Transl Cancer Res 2021;10(2):779-789. doi: 10.21037/tcr-20-2560 advanced lung adenocarcinoma. Tumour Biol 2018;40:1010428318760420.

14. Lang D, Horner A, Brehm E, et al. Early serum tumor marker dynamics predict progression-free and overall survival in single PD-1/PD-L1 inhibitor treated advanced NSCLC-A retrospective cohort study. Lung Cancer 2019;134:59-65.

15. Cai Z, Chen G, Zeng Y, et al. Comprehensive Liquid Profiling of Circulating Tumor DNA and Protein Biomarkers in Long-Term Follow-Up Patients with Hepatocellular Carcinoma. Clin Cancer Res 2019;25:5284-94.

16. Hodi FS, Hwu WJ, Kefford R, et al. Evaluation of Immune-Related Response Criteria and RECIST v1.1 in Patients With Advanced Melanoma Treated With Pembrolizumab. J Clin Oncol 2016;34:1510-7.

17. Cyriac G, Gandhi L. Emerging biomarkers for immune checkpoint inhibition in lung cancer. Semin Cancer Biol 2018;52:269-77. 
Supplementary

Table S1 Specific serum tumor markers and reference ranges for various malignant tumors

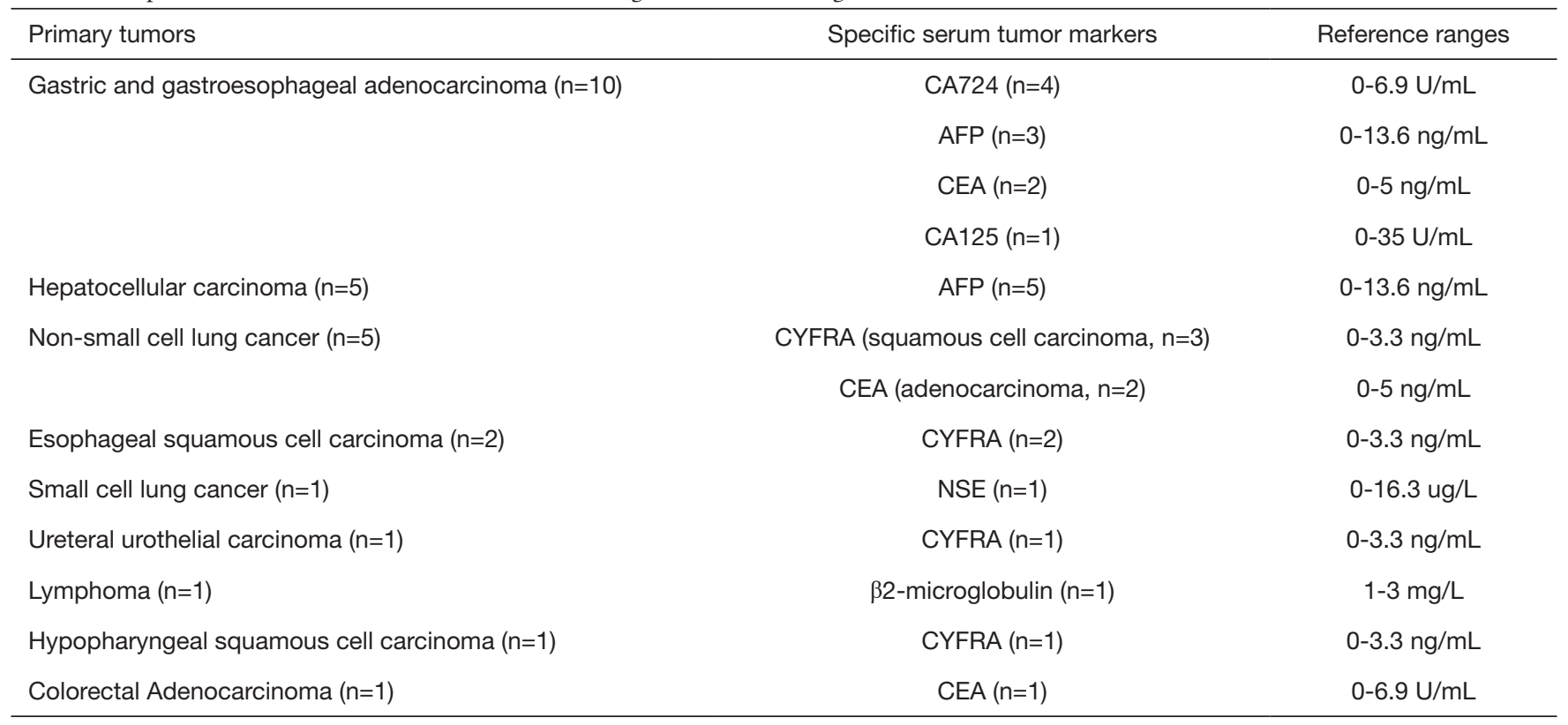

CA724: carbohydrate antigen 724; AFP: alpha-fetoprotein; CEA: carcinoembryonic antigen; CA125: carbohydrate antigen 125; CYFRA: cytokeratin fragment; NSE: neuron-specific enolase. 